\title{
El concepto de selección interna y la sujeción de la Biología Evolucionaria del Desarrollo al modelo de explicación variacional
}

\author{
Gustavo Caponi \\ Departamento de Filosofia, CFH, Universidade Federal de Santa Catarina \\ Caixa Postal 476, CEP 88010-970, Florianópolis - SC, Barsil \\ gustavocaponi@newsite.com.br
}

Submetido em 02/04/2008

Aceito para publicação em 08/08/2008

\section{Resumo}

O conceito de seleção interna e a sujeição da Biologia Evolucionaria Desenvolvimental ao modelo de explicação variacional. A idéia de seleção interna, proposta originalmente por Lancelot Whyte, não só serve para entender o estatuto causal das constrições desenvolvimentais, mas também nos permite compreender como esses fatores organísmicos ou internos, cuja importância a Biologia Evolucionaria Desenvolvimental hoje quer resgatar, podem ser considerados desde uma perspectiva variacional ou seleccional compatível, mas não assimilável, à Teoria da Seleção Natural. Assim, considerado como um conceito autônomo e diferente ao de seleção natural, o conceito de seleção interna faz possível compreender de que modo isso que Wallace Arthur chama viés desenvolvimiental constitui uma causa, efetiva e positiva, de transformações evolutivas irreversíveis e acumuláveis.

Unitermos: Arthur, W., Whyte, L., seleção interna, seleção natural, viés desenvolvimental

\section{Abstract}

The concept of internal selection and the subjection of Evolutionary Developmental Biology to the variational model of explanation. The idea of internal selection, originally proposed by Lancelot Whyte, not only helps us to understand the causal status of developmental constraints, but can also show us how these organismic or internal factors, whose relevancy Evolutionary Developmental Biology highlights today, can be considered from a variational or selectional perspective that is compatible with, but not reducible to Natural Selection Theory. Thus, since it is considered as a concept that is autonomous and different from natural selection, the concept of internal selection can enable us to understand how the phenomenon that Wallace Arthur calls developmental bias constitutes an effective and positive cause of cumulative and irreversible evolutionary changes.

Key words: Arthur, W., Whyte, L., internal selection, natural selection, developmental bias

\section{Presentación}

Propuesto por Lancelot Law Whyte (1960a; 1960b; 1964 y 1965) en la primera mitad de los años sesenta, el concepto de selección interna fue largamente ignorado en la Biología Evolucionaria. Recientemente, sin embargo, algunos autores comprometidos con la construcción de la Biología Evolucionaria del Desarrollo, comenzaron 
a rescatarlo como una clave para explicar la manera por la cual los fenómenos ontogenéticos inciden en el curso de la evolución (por ejemplo: Arthur, 1997 y 2004a; Wagner y Schwenky, 2000 y 2003). La selección interna puede, en efecto, explicar ese sesgo en la oferta de variaciones que, en los últimos tiempos, está siendo reconocido como una causa rectora de los fenómenos evolutivos equiparable, por su importancia, a la selección natural (Arthur, 2001 y 2004b); y es en ese sentido que cabría pensarla como el concepto fundamental, o como la clave ordenadora, de la Biología Evolucionaria del Desarrollo.

No es esa, por lo general, la forma en que el concepto es considerado por los pocos biólogos evolucionarios que apelan a él para explicar algún que otro caso de constreñimiento ontogenético de la evolución; pero, creo que se puede mostrar que su importancia sistemática trasciende esos usos más restringidos. La idea de selección interna no sólo sirve para entender mejor el modus operandi de dichas constreñimientos ontogenéticos sino que además puede hacernos comprender de qué modo esos factores orgánicos o internos, cuya importancia la Biología Evolucionaria del Desarrollo hoy quiere rescatar, son pasibles de ser considerados desde una perspectiva variacional o seleccional (Sober, 1984; Lewontin, 2000) afín y compatible con la Teoría de la Selección Natural. Pero digo compatible para no decir asimilable: el concepto de selección interna, según también espero mostrar, no es reducible al de selección natural; y su autonomía puede permitirnos comprender por qué eso que Wallace Arthur llama sesgo del desarrollo [developmental bias], constituye una causa de transformaciones evolutivas, efectiva y positiva, que es independiente de la propia selección natural (Arthur, 2001; 2004a; 2004b y 2006).

Para tanto, procederé primero a analizar el concepto de selección interna conforme fue presentado por Lancelot Whyte. Insistiré, a continuación, en sus diferencias con el concepto darwiniano de selección natural; y luego intentaré mostrar cómo este concepto puede servirnos para entender aquello que Wallace Arthur llama sesgo ontogenético. A continuación mostraré cómo los análisis de Whyte sirven para esclarecer de qué modo lo que Pere Alberch (1989) y
Stephen Jay Gould (2002) 1lamaron internalismo puede ser considerado desde una perspectiva variacional; y por último intentaré dar algunas indicaciones sobre cómo la selección interna puede ser considerada la causa de un proceso irreversible de cambios acumulativos capaces de facilitar la acción de la propia selección natural. Es importante subrayar, sin embargo, que esta débil incursión en el dominio de la especulación científica sólo tiene el objetivo de subrayar cómo el recurso al concepto de selección interna permite entender de qué manera los factores ontogenéticos, o internos, son pasibles de ser pensados como causas efectivas del cambio evolutivo relativamente independientes de la selección natural.

Creo importante subrayar, por otro lado, que mi objetivo no es el de rehabilitar un autor injustamente olvidado por la cruel ortodoxia neodarwiniana. Este retorno a las tesis de Whyte sólo se justifica porque, según me parece, el concepto de selección interna facilita el análisis epistemológico de los desarrollos de la Biología Evolucionaria del Desarrollo. El mismo, creo, nos permite mostrar de un modo claro qué es lo que hay de peculiar en el decir de ese nuevo capítulo de los estudios evolucionarios.

La analogía entre las tesis de Whyte y aquellas defendidas por los cultores de la Biología Evolucionaria del Desarrollo es, después de todo, bastante evidente; y reparar en ellas no constituye ningún hallazgo: Wallace Arthur (2004a) ya lo ha hecho. La insistencia de Whyte (1965) en la tesis de que "[no]todas las propiedades de los organismos son consecuencia de la selección adaptativa previa" y su afirmación de que "muchas propiedades, especialmente las de coordinación, pueden ser el resultado de la selección interna", se corresponde perfectamente con la tesis según la cual muchos rasgos de los seres vivos pueden ser producto de requerimientos intrínsecos a la dinámica ontogenética y no respuestas a presiones selectivas presentes o pasadas. La afirmación de que "las condiciones de la organización y el desarrollo necesariamente imponen restricciones sobre las posibles líneas del cambio evolutivo"(Whyte, 1965) parece extraída de un manual de Biología Evolucionaria del Desarrollo; y los constraints a los que esos textos tanto aluden no parecen nada diferente de esas restrictions de las que hablaba Whyte. 


\section{Integración y Desarrollo}

Después de haberlo esbozado en dos breves artículos (1960 y 1964), Whyte desarrolló el concepto de selección interna en su opúsculo de 1965 titulado Internal factors in evolution; y ya en el prefacio decía que la idea central de ese trabajo era sostener que "además de la selección darwiniana otro proceso también había jugado un rol importante en la determinación de la evolución de las especies". Es decir: "al lado de la bien establecida selección competitiva exterior de la teoría 'sintética' de la evolución”, había también "un proceso de selección interna actuando directamente sobre las mutaciones, principalmente en los niveles molecular, cromosómico y celular, pero no en términos de lucha y competición, sino en función de la capacidad sistémica para una actividad coordinada"; y, por eso, continuaba Whyte, "el criterio darwiniano de aptitud para la competición externa" debía ser suplido, no suplantado, por "el de la buena coordinación interna". En los seres vivos, consideraba correctamente Whyte, "la coadaptación interna es tan necesaria cuanto la adaptación externa"; y era necesario considerarla a la hora de explicar la evolución (Whyte, 1965; ver también: Arthur, 2004a).

En realidad, Whyte estaba haciendo referencia a una cuestión que ya había sido planteada por otros autores que se sentían incomodados por el descuido con el que el darwinismo trataba la exigencia de coordinación internas de las partes que Cuvier (1992 [1812]) había destacado en su Principio de la Correlación de las Formas en los Seres Organizados: "todo ser organizado forma un conjunto, un sistema único y cerrado, en el cual todas las partes se corresponden mutuamente, y concurren a la misma acción definitiva por una acción reciproca". Tal era el caso, por ejemplo, de Edward Stuart Russell (1916) que, en su célebre Form and Function había criticado la escasa importancia que Darwin le concedía a la necesaria “correlación de las variaciones". Pero también se podría citar a Jakob Von Uexküll (1945 [1913]) cuando, más o menos para la misma época, acusaba al darwinismo de hacernos olvidar que, además de estar "adaptado conforme a fin a su entorno", un organismo también "está construido conforme a fin en sí mismo".

Claro, Whyte ya está muy lejos de los años de eclipse del darwinismo (Huxley, 1943; Bowler, 1985).
Él escribe, incluso, en ese periodo en el que ocurre lo que Gould (2002) caracterizó como un endurecimiento de la Nueva Síntesis; y sus argumentos asumen los logros de la Teoría de la Selección Natural sin perderse en diatribas antidarwinistas (cf. Whyte, 1965). Pero, aun así, su propuesta para complementar esos logros se funda en un organicismo afín a las posiciones de autores como Ludwig Von Bertalanffy (1979 [1953]) y Kurt Goldstein (1951), a los que Whyte (1965) incluso cita como precedentes de sus posiciones. Y ese compromiso con el organicismo se patentiza en su insistencia en el hecho de que cualquier cambio en las estructuras y funciones de todo ser vivo debía adecuarse a lo que él llamaba Condiciones Coordinativas $(C C)$; es decir: "las reglas de orden que deben ser satisfechas por las partes internas y los procesos de cualquier organismo celular capaz de desarrollarse y de sobrevivir en algún ambiente" (Whyte, 1965, itálicos míos).

Pero, si con todo derecho, estas Condiciones Coordinativas nos recuerdan al Principio de las Condiciones de Existencia (Cuvier, 1817), tomado en su sentido originario, cuvieriano, de coherencia funcional, y no en el sentido darwiniano de adecuación a las condiciones de vida (cf. Russell, 1916; Grene y Depew, 2004; Caponi, 2005); debemos también subrayar que la tesis de Whyte no sólo alude a la correlación de las partes en el organismo ya constituido, sino también, y sobre todo, a la coordinación y coherencia de las distintas etapas de su ontogénesis. Las $C C$, nos dice Whyte (1965): "no son meramente morfológicas [...], sino también morfogenéticas"; y, en su opinión, este aspecto ontogenético es más importante que el meramente funcional (Whyte, 1965). Para él, en efecto, "el desarrollo es teóricamente anterior a la homeostasis" (Whyte, 1965). No es, sin embargo, el primado de la ontogenia sobre la homeostasis lo que más debe preocuparnos aquí.

En lo que respecta a la Biología Evolucionaria, la tesis más provocativa y relevante de Whyte no está ahí. Ella reside, más bien, en la presunción de que la ontogenia es teóricamente anterior a la filogenia (Whyte, 1965); y esa prioridad es muy fácil de entender si se acepta que la filogenia no es más que "una secuencia de relatos ontogenéticos" (Whyte, 1965). Es obvio, además, 
que "el crecimiento de un individuo puede ser descrito, en principio, sin considerar la evolución de las formas de vida, pero la evolución no tiene sentido si no hay individuos que hayan crecido"; por eso: "el desarrollo ontogenético debe ser entendido antes que los cambios históricos en ese desarrollo"(Whyte, 1965). Whyte aceptaba, en efecto, eso que Ron Amundson (2005) recientemente ha llamado Principio de Completud Causal: "Para producir una modificación en la forma adulta, la evolución debe modificar el proceso embriológico responsable por esa forma. Por eso, para comprender la evolución es necesario comprender el desarrollo"; y esto es crucial para entender cómo es que "la estructura interna de los organismos ha influenciado directamente las avenidas de la filogenia" (Whyte, 1965).

Como sabemos, la materia prima sobre la que trabaja la selección natural es la variación hereditaria; y esto Whyte no lo ponía en duda. Pero, conforme él insistía, para que esas variaciones pudiesen producir fenotipos capaces de entrar en el juego de la competición darwiniana, es menester que las mismas produzcan cambios compatibles con las Condiciones Coordinativas. Éstas, "al igual que una ecuación algebraica" pueden ser satisfechas "por muchas soluciones particulares" (Whyte, 1965); pero, si una variación hereditaria produce una reformulación de la ontogenia, ésta debe ser tal que genere una nueva solución [correcta] de dicha ecuación: ella debe ser "un cambio desde una solución particular de las CC hacia otra". Por eso, observaba Whyte, las $C C$ pueden "revelar por qué el curso de la evolución despliega una serie de tipos estructurales que se siguen las unos a los otros en una determinada secuencia necesaria". Esos tipos, y sus formas particulares, nos muestran las diferentes soluciones posibles de las $C C$; y su secuencia evolutiva nos indica el orden en que esos cambios deben ocurrir para que, en cada etapa, se produzcan alteraciones que no rompan con esas Condiciones Coordinativas.

Usando un lenguaje más moderno, se podría también decir que las $C C$ no sólo predefinen el morfoespacio que la evolución habrá eventualmente de ocupar; sino que además predeterminan la secuencia en la cual ese morfoespacio podrá ser ocupado. Así, partiendo de un punto determinado en ese morfoespacio, "las condiciones de la organización biológica restringen a un espectro finito y discreto las posibles avenidas del cambio evolutivo"; y es atendiendo a esto que Whyte afirma que "la selección adaptativa darwiniana no ha sido el único agente director que guió la evolución de las formas vivas por los corredores que ella efectivamente siguió". "La naturaleza de la vida, el propio carácter estructural de los organismos, impuso restricciones básicas en los cambios que son permisibles"; y por eso esas exigencias organizacionales deben ser reconocidas como "un factor que dirige a la filogenia" conjuntamente con esa fuerza más conocida que Darwin llamó selección natural.

Es decir: "mientras en la teoría sintética se afirma que el único factor directivo en la filogenia es la selección externa darwiniana que actúa sobre formas maduras en virtud de su eficacia reproductiva en ambientes particulares, ahora un segundo factor directivo ha emergido: los prerrequisitos de un estricto orden interno expresados en las $C C$. La vida sólo puede evolucionar dentro de las anchas avenidas determinadas por su propia naturaleza estructural" (Whyte, 1965). Claro, "las trayectorias efectivamente seguidas dentro de esas avenidas son determinadas por la selección externa darwiniana", pero es un "previo proceso selectivo interno, más que la selección darwiniana, el que asegura que sólo genotipos bien integrados sobrevivan" (Whyte, 1965); y es a eso que Whyte llama de selección interna: un conjunto de "procesos selectivos que actúan directamente sobre las consecuencias iniciales del genotipo que aseguran que las $C C$ son satisfechas por todos los tipos mutantes que sobreviven hasta el punto en el cual la selección externa darwiniana opera" (Whyte, 1965).

Inicialmente, en el glosario, también se había usado la expresión developmental selection (Whyte, 1965); y la misma nos parece definitivamente más adecuada que selección interna. Pero, para reforzar el contraste con la selección externa o darwiniana, Whyte, que se resiste a caracterizar a esta última como siendo la única natural, acaba prefiriendo el calificativo de interna. De todos modos, y más allá de esta cuestión terminológica, lo concreto es que en ese glosario se formulan dos definiciones complementarias, $\mathrm{y}$ altamente 
esclarecedoras, de esta nueva fuerza evolutiva. En la primera, la selección interna aparece descripta como selección "de mutantes, en los niveles molecular, cromosómico y celular, en virtud de su compatibilidad con la coordinación interna del organismo" (Whyte, 1965); y en la segunda se la caracteriza como "la restricción de las direcciones hipotéticamente posibles del cambio evolutivo por factores organizacionales internos" (Whyte, 1965).

En el primer caso, podríamos decir, la selección interna aparece caracterizada como una causa próxima que actúa en el proceso de constitución de cada organismo individual; y en el segundo ella es entendida como una causa remota cuyos efectos se verifican en la filogenia. Pero, aunque Whyte (1965) acabe privilegiando esta última acepción, lo cierto es que ambas definiciones son necesarias para entender sus tesis. Del mismo modo en que no puede haber selección natural sin lucha por la existencia, las $C C$ tampoco podrían pautar la evolución si sus exigencias no se hiciesen sentir abortando, y tal vez revirtiendo o reconfigurando, como Whyte también pretendía, cada proceso ontogenético individual que venga a transgredirlas. Y aquí es oportuno resaltar que Whyte distingue tres mecanismos diferentes de selección interna.

El primero es la reversión de las configuraciones moleculares inapropiadas, el segundo es la transformación de esas configuraciones inapropiadas en otras que satisfagan las condiciones coordinativas; y el tercero es la simple eliminación de las malas configuraciones (Whyte, 1965). Tal vez, las dos primeras hoy pueden ser asociadas a los mecanismos de reparación del ADN. Mecanismos que, como dice Evelyn Fox Keller (2000), al "sacrificar la fidelidad en vistas a garantizar la continuidad del proceso de replicación", se transforman en factores productores de variaciones apropiadas que se parecen, sobre todo, a las reconfiguraciones coordinativamente correctas postuladas por Whyte.

Pero, más allá de que las conjeturas de Whyte sobre la existencia de tales mecanismos hayan estado, o no, bien encaminadas y hayan sido de algún modo confirmadas por los desarrollos posteriores de la Biología Molecular, creo que esos dispositivos de reparación son substancialmente diferentes de la simple eliminación de las configuraciones contrarias a las condiciones coordinativas; y lo son por la sencilla razón de que, estrictamente hablando, esas correcciones no conforman un mecanismo selectivo. Ellas, de hecho, sólo ayudan a componer la oferta de variantes sobre la que siempre acaba actuando el tercero de los mecanismos previstos; y es ése, el eliminativo, el que tendremos en cuenta en este trabajo: será a él al que siempre nos referiremos con el término selección interna. Los otros dos, además, podrían de hecho no existir; y, aun así, ese tercer e inevitable mecanismo de eliminación podría continuar ejerciendo su control sobre las configuraciones de los seres vivos.

\section{Selección Interna y Selección Natural}

Se podría objetar, como Lewontin y Caspari (1960) lo hicieron, que la expresión selección interna, sobre todo si es tomada en el sentido restringido aquí propuesto, puede no ser otra cosa que un nuevo rótulo para la selección normalizante: esa forma particular de selección natural que, al decir de Dobzhansky (1983), "deja el trasfondo génico limpio de variantes genéticas incondicionalmente deletéreas, o, por lo menos, reduce sus frecuencias a un mínimo irreductible"(ver también: Dobzhansky et al., 1980). Creo, sin embargo, que ambos conceptos, el de selección interna y el de selección normalizante pueden, y deben, ser distinguidos. Dejaré esa cuestión, sin embargo, para un poco más adelante y me centraré primero en la distinción entre selección interna y selección natural darwiniana o externa en general. A primera vista, la misma puede parecer una distinción arbitraría y se hace necesario insistir sobre la diferencia cualitativa que existe entre ambos conceptos. La tal vez tenue distinción entre selección interna y selección normalizante será sólo un corolario de las claras diferencias que existen entre selección interna y selección natural en general.

En contra de lo que Lewontin y Caspari sugerían (1960), no se deben subestimar las importantes diferencias que existen entre ambas nociones. Pero eso es lo que Wallace Arthur (2004a) de hecho hace en Biased embryos and evolution cuando dice que "probablemente no existe una cosa tal como una pura selección externa y una pura selección interna" y que 
"si estos fenómenos existen, ellos no son más que los extremos opuestos de un espectro continuo". Posición que contrasta con aquello que él mismo había dicho en The origin of animal body plans. Allí, siguiendo más de cerca a las tesis de Whyte, Arthur (1997) había escrito que "en términos de causalidad biológica, la selección interna es claramente diferente de la selección externa. No hay un continuo de una a la otra [...]. Un agente selectivo es o bien parte del organismo o bien no lo es". Y esa es, de hecho, la posición sustentada por Whyte. Éste, conociendo ya esa posible confusión entre ambos conceptos (cfr. Whyte, 1960b y 1965) había resaltado la diferencia entre ambos diciendo:

La selección adaptativa darwiniana es un proceso externo relativo, estadístico, efectivo entre pares de individuos o poblaciones, un asunto de grado determinado sólo por aptitud comparativa y frecuencias en algún ambiente particular. La selección interna es proceso intrínseco, usualmente de todo o nada, operando dentro de individuos aislados, y que puede ser determinado por la simple observación de la historia de un organismo en el ambiente más favorable. La selección adaptativa depende de un alto índice de mortalidad y expresa competición; la selección interna se basa en la coordinación dentro de los individuos (Whyte, 1965).

La selección natural darwiniana, en suma, es un proceso poblacional que supone la competición, y el éxito reproductivo diferencial, comparable, de alternativas fenotípicas con el mínimo de viabilidad, o de competitividad, requerido para participar efectivamente en esa pugna. La selección interna, en cambio "se evidencia en la historia estructural de los organismos", y dado que "muchos tipos [por ella] excluidos nunca ingresan en estadísticas poblacionales" (Whyte, 1965) sus efectos no pueden verificarse en los parámetros previstos por la genética de poblaciones. No cabe afirmar, en este sentido, que en los modelos de la genética poblacional "los agentes de la selección interna y externa" sean, como dice Arthur (1997) "formalmente indistinguibles". Lo cierto, en todo caso, es que esos modelos son inadecuados para captar la acción de la selección interna; por eso, y como ya decía Whyte (1965): "la teoría estadística de las poblaciones debe ser complementada por una teoría estructural de la ontogénesis individual y de su influencia sobre la filogenia".
Malgré Günter Wagner y Kurt Shwenck (2003), la eliminación de una variación que produce una disrupción de la dinámica ontogenética tal que causa la muerte del embrión antes de que éste entre en la población, sería un fenómeno que nunca podría ser considerado como un caso de selección natural. Ese sería, por el contrario, un perfecto ejemplo de selección interna; es decir: sería un ejemplo de la autoeliminación sumaria a la que está condenado todo aquello que, no llegando a cumplir con los requisitos de las $C C$, tampoco cumple con los requerimientos mínimos que son necesarios para entrar en esa competición que es la lucha por la existencia. Tampoco puede decirse, por eso, que "las variantes que producen una disrupción en la dinámica interna de los sistemas funcionales u ontogenéticos tengan consecuencias negativas para la aptitud de un organismo" (Wagner y Shwenck, 2003). Aunque, a primera vista, esto pueda parecer algo totalmente razonable, casi la descripción de un hecho obvio, en realidad se trata de un craso error categoríal provocado por la violación subrepticia de la gramática que sanciona el buen uso del concepto de aptitud.

Es ese error el que hace que Günter Wagner y Kurt Shwenck (2003) consideren a la selección interna y a la selección externa como dos componentes de la selección natural. Para ellos, en efecto, "la distinción crucial" entre ambos conceptos reside simplemente en el hecho de que "la selección externa cambia con el ambiente, mientras la selección interna permanece esencialmente constante porque ella viaja con el organismo" (Wagner y Shwenck, 2003); y aunque esta formulación parezca bastante feliz la misma encubre un hecho clave: la selección interna no produce diferencias de aptitud. "La selección interna", ya lo decía Whyte (1965), "no actúa causando supervivencia diferencial de dos tipos competitivos, sino que acepta o rechaza cada genotipo mutado por sí mismo. El San Pedro de la selección interna no hace comparaciones odiosas, sino que juzga a cada candidato en virtud de sus propios méritos".

Mientras tanto, y diferentemente de la adecuación a las condiciones coordinativas, la aptitud es una magnitud comparativa que indica el éxito reproductivo diferencial de una variante al interior de una población; y por eso la aptitud de un fenotipo puede llegar a incrementarse con 
el simple decrecimiento de la aptitud de su alternativa competidora. Cosa que jamás ocurriría con una variante letal: por más que la aptitud de la variante nodisruptiva disminuya, la aptitud de la forma letal nunca se incrementará. Se podría hablar aquí, de una aptitud nula; pero es importante resaltar que para establecer esa nulidad no precisaríamos de las comparaciones supuestas en el concepto darwiniano de aptitud: nada precisaríamos saber sobre el éxito reproductivo de la variante no-letal para determinar que aquella que aborta el proceso de desarrollo carece de toda aptitud posible. Es decir: mientras la acción de la selección natural sólo puede registrarse comparando la desigual frecuencia relativa de las alternativas en pugna, la acción de la selección interna puede ser registrada analizando un único ciclo vital.

"La evidencia de la selección interna", ya lo decía Whyte (1965) con toda claridad, "no es una tasa relativa de aumento en un ambiente dado, sino la observación estructural directa de la coordinación interna exitosa (o fallida) de un único organismo mutante". Es cierto: todo proceso de desarrollo ocurre, y supone, un cierto entorno; y lo que sería letal y disruptivo en un entorno podría no serlo en otro. Una cierta reprogramación ontogenética puede ser letal para un animal anfibio y podría no serlo para uno acuático. Pero, en ese caso, no podría decirse que esa variante contradice las condiciones coordinativas. Ella las satisface puesto que de hecho existe un contexto posible en el cual la reprogramación ontogenética que ella genera se torna viable; y eso puede darnos una clave para distinguir los conceptos de selección normalizadora y selección interna. Éste aludiría a las exigencias más generales y universales de las condiciones coordinativas y aquél se referiría a lo que, aun siendo letal, y totalmente incompetente, en el ambiente ocupado por la población en estudio, podría gozar de cierta aptitud en otros ambientes tal vez muy particulares y restrictos.

La selección interna tiene que ver con lo siempre incompetente: tiene que ver con aquello que nunca podrá encontrar el ambiente el cual gozar de un mínimo éxito reproductivo; y esto se puede deber a dos tipos de razones: a la total inviabilidad de la ontogenia o a su carácter irremediablemente inconcluso. La selección interna, como lo dice el propio Whyte (1965), no sólo incluye la eliminación de los genotipos que generan embriones o larvas inviables; sino que también tiene que ver con esas variaciones hereditarias que "matan al individuo antes de la madurez o lo dejan infértil". Los organismos son ciclos vitales y una de las etapas cruciales de todo ciclo vital es aquella en la que se adquiere la aptitud para la reproducción (Arthur, 2006). Un ser que, por su propia dinámica ontogenética, llegue a la senescencia y a la muerte sin nunca poder pasar por esa fase, no satisfaría las condiciones coordinativas y, por consecuencia, sería invisible para la selección natural.

Si el pichón que está formándose en un huevo tiene condiciones funcionales de llegar a su edad reproductiva, aunque sea protegido en el ambiente artificial de un laboratorio: él será considerado como acorde a las $C C$. Incluso cuando toda la evidencia nos indique que, en la naturaleza, ni siquiera hubiese podido preservar su lugar en el nido por unas pocas horas. Por el contrario, un búfalo portador de una mutación que afecta su sistema endocrino al punto de hacerlo mayor y más fuerte que sus semejantes, pero que al mismo tiempo lo deja irremediablemente estéril, no estaría satisfaciendo las $C C$; y, por eso, pese a su adaptación ecológica individual superior, jamás podría entrar en competición darwiniana con sus congéneres más pequeños y débiles por el simple hecho de nunca alcanzar esa fase crucial de la ontogenia que es la edad reproductiva.

En síntesis: dado el universo de todas las condiciones ecológicas posibles en las que un proceso ontogenético puede completarse, desde las más benignas a las más exigentes, desde las más habituales a las más improbables, las condiciones coordinativas son satisfechas por cualquier proceso ontogenético que pueda llegar a su etapa reproductiva aunque sea en un subconjunto muy restricto e improbable de dichas condiciones ecológicas. Y es esto lo que Arthur (2004a) parece pasar por alto cuando pretende establecer un contraste entre la supuesta selección [puramente] externa que podría beneficiar un incremento de tamaño en ciertas moscas y la supuesta selección [casi exclusivamente] interna que acabaría castigando cualquier mutación que implique una articulación defectuosa entre las alas y el tórax de dichas moscas. 
En lo primero, claro, Arthur no se equivoca: dentro de una población de moscas sometidas a una disminución de la humedad ambiente, un incremento del tamaño, como él bien observa, puede representar una ventaja adaptativa, en tanto y en cuanto ese mayor volumen implica una disminución relativa de la superficie corporal en contacto con el aire (Arthur, 2004a). En ese caso, las moscas mayores, si eso nos las torna, por ejemplo, más fáciles de ser cazadas, tendrán una aptitud superior a las menores; y esta diferencia de aptitud estará obviamente correlacionada con un factor ambiental que, de revertirse, podrá volver a tornar más aptas a las moscas menores. Estaríamos ahí ante un clásico ejemplo de selección natural darwiniana, o selección externa, beneficiando ciertas formas alternativas en detrimento de otras; y la presión selectiva en juego tendría que ver con un factor claramente ambiental (Arthur, 2004a).

Pero, creo que Arthur (2004a) no razona de modo correctamente darwiniano, ni tampoco razona como Whyte lo haría, cuando dice que una variación hereditaria que disminuya la articulación entre las alas y el tórax de esas moscas, disminuyendo su capacidad para volar, sería adaptativamente inconveniente en cualquier ambiente; $y$, por lo tanto, su eliminación selectiva podría ser considerada como un caso de selección interna a la Whyte. En esa situación, nos dice Arthur (2004a), "las diferencias de aptitud entre individuos no son causadas por ninguna característica específica del ambiente": ellas serían, más bien, ambiente-independientes [environmentindependent] o casi ambiente-independientes. Pero ahí se esconden dos errores: el primero consiste en dar por descontado que, "aunque el vuelo ocurre en el ambiente en un sentido general, la habilidad para el buen vuelo no es solamente una ventaja en cualquier selva o pradera en particular; sino que ella lo es en todas las selvas y praderas"(Arthur, 2004a); y el segundo consiste en suponer que, por esa razón, la selección que estaría ocurriendo en contra de esa característica sería "la selección interna de Whyte"(Arthur, 2004a).

Claro, imaginar un ambiente, o condiciones de vida, en donde las moscas no precisen volar, no es muy fácil. Es casi tan difícil como imaginar un ambiente en donde un animal semejante a una musaraña no precise de una visión minimamente desarrollada; $y$, sin embargo, ahí están los topos que, de hecho, son descendientes de alguna forma primitiva de musaraña (Lecointre y Le Guyader, 2001). Las condiciones de vida a las que pueden estar sometidos los seres vivos son tan variadas, y las formas en que los recursos del ambiente pueden ser explotados, son tan imprevisibles, que nada nos puede permitir afirmar que una morfología ontogenéticamente producible, como parece serlo la de la mosca mal alada, no pueda nunca encontrar, o producir, un nicho en el cual esa característica resulte en una ventaja. En una región muy árida, pero tal vez repleta de pequeñas cavernas oscuras y húmedas en donde ciertas moscas depositan sus larvas, de pronto podría surgir una variante de las mismas que encuentre ventaja permaneciendo en una vida subterránea lejos del sol y del aire seco; y pocas moscas estarían mejor preparadas para eso que aquellas que la falta de buenas alas, al impedirles volar hacia un exterior deshidratante, las predisponga a deambular por esas pequeñas galerías subterráneas.

Es verdad que esas circunstancias ecológicas pueden no darse jamás. Pero eso tampoco implica que no sea la selección natural la que presiona en contra de los mutantes de alas mal articuladas. Si en una población de moscas se atenuasen las exigencias que hacen del vuelo una necesidad adaptativa - pensemos, por ejemplo, en una disminución de la depredación simultanea con un aumento de las fuentes de alimentos explotadas por las moscas-, la frecuencia de los individuos de alas mal articuladas podría incrementarse aun cuando nunca llegue a superar la frecuencia de los individuos normales; y eso nos estaría indicando que, en las circunstancias habituales, era la propia selección natural darwiniana, y no la selección interna, la que impedía que esa frecuencia se incrementase. Es claro, además, que de volver a imperar esas circunstancias antes habituales, depredación más intensa y menor abundancia de alimentos, sería también la selección natural la que haría retornar esas frecuencias relativas a sus niveles anteriores.

\section{La selección interna como productora del sesgo ontogenético}

Una constreñimiento ontogenético, conforme fue definido en el consensus paper organizado por Maynard Smith, Richard Burian y Stuart Kauffman en 1985, sería 
"un sesgo en la producción de variantes fenotípicas o una limitación de la variabilidad fenotípica, causada por la estructura, carácter, composición, o dinámica del sistema en desarrollo"(Maynard Smith et al., 1985); y, para entender esos constreñimientos, debemos pensar en lo siguiente: toda innovación evolutiva posible, toda variación que pueda ofrecerse al escrutinio de la selección natural, tiene que poder corporizarse antes en una alteración ontogenética viable (Amundson, 2001; Schwenk y Wagner, 2003). Es decir: tiene que corporizarse en un ciclo ontogenético capaz de satisfacer las coordinaciones coordinativas a las que se refería Whyte. Recordando, claro, que éstas no sólo se refieren a la coordinación de las partes en cualquier etapa particular de la ontogenia; sino también, y sobre todo, a la propia congruencia entre dichas etapas.

Sea cuál sea la índole de la variación fenotípica que venga a ser sometida al veredicto de la selección natural, y sea cuál sea ese veredicto, ella tiene que cumplir, antes, con dos requisitos fundamentales: en primer lugar ella tiene que ser accesible para el sistema en desarrollo (Maynard Smith et al., 1985; Raff, 2000), es decir: tiene que tratarse de una alteración pasible de ser producida en y por ese mismo proceso ontogénetico (cf. Arthur, 1997; Azkonobieta, 2005); y, en segundo lugar, ella tiene que ser tal que, ni aborte ese proceso, ni genere un monstruo totalmente inviable (Amundson, 2001). Un cambio evolutivo tiene que ser ontogenéticamente posible (Amundson, 2005; Azkonobieta, 2005); y, por eso, aun cuando la ontogénesis pueda o no recapitular a la filogénesis, debemos reconocer que, con seguridad, la limita y la orienta (Hall, 1992; Wilkins, 2002) tal y como Lancelot Whyte lo pretendía.

La limita estableciendo cuáles modificaciones morfológicas y funcionales son viables y cuáles no; pero al hacer eso también la orienta: si un rasgo $a$ puede cambiar a la forma $a^{1} \mathrm{o}$ a la forma $a^{2}$, pero la viabilidad de $a^{1}$ depende de que simultáneamente a ella se dé otra serie compleja de cambios en otros rasgos, y la viabilidad de $a^{2}$ no depende de esa coincidencia feliz; entonces éste será un cambio más probable que aquél. Para la evolución, para decirlo de algún modo, el estado $a^{2}$ será más accesible que el estado $a^{1}$; y esto puede explicar que $a^{2}$ se dé, y no $a^{1}$, aun cuando nosotros pudiésemos imaginar que éste sería darwinianamente más eficaz que aquél. Una innovación puede ser muy útil pero si es muy difícil de ser incorporada al proceso de desarrollo, si el cambio en la solución de las condiciones coordinativas exige demasiados pasos; entonces es muy posible que dicha innovación nunca ocurra y que en su lugar ocurra otra, tal vez un poco menos eficaz en términos adaptativos, pero que exige una reformulación menor y menos improbable, más fácil diría Wallace Arthur (2004a y b), de la ontogénesis.

Por otro lado, además de permitirnos explicar la oferta de variaciones que pueden darse en una determinada población, el estudio de los procesos de desarrollo también puede permitirnos entender la secuencia de las innovaciones evolutivas. La ontogénesis es un proceso necesariamente secuencial: una estructura solo surge cuando existen otras estructuras previas que le sirven de base; y el estudio de esas etapas puede permitirnos determinar cuáles fueron los pasos que siguió la propia evolución. Si en la ontogénesis, un órgano A surge por la diferenciación de las células que componen el tejido de un órgano B ya parcialmente conformado; entonces, podemos inferir que A es una innovación evolutiva posterior a $\mathrm{B}$. La filogénesis puede hacer muchas cosas con A y con B; pero si las células de A son una especialización de las células de $\mathrm{B}$, ella necesariamente tuvo que producir a $\mathrm{B}$ antes que a $\mathrm{A}$. La ontogénesis, lo vemos otra vez, pauta y ordena los pasos de la filogénesis (Hall, 1992; Amundson, 2005); y es por eso que Wallace Arthur (2001 y 2004b) tiene razón al insistir en el hecho de que el sesgo ontogenéticos produce tanto constreñimientos cuanto direccionamientos.

La selección natural, claro, habrá de ser siempre el tribunal que juzgará la conveniencia y la oportunidad ecológica de cada una de esas innovaciones; pero ella no podrá alterar su secuencia. Y esto es muy importante para entender y reconstruir las sendas efectivamente seguidas por la evolución. La variación posible de la información hereditaria puede ser isotrópica como Wallace (1891) quería; pero, de hecho, la oferta de alternativas a ser seleccionadas ciertamente no lo es (Arthur, 1997 y 2004a). Entre la variación genética y la selección natural opera otro filtro: aquél que discrimina entre alteraciones viables y alteraciones no viables de 
la ontogénesis; y es a ese filtro precisamente que alude el concepto de selección interna propuesto por Whyte. Lo difícil, claro, es comprender cómo es que ese primer filtro funciona (Arthur, 1997 y 2004a).

Individualizar y cartografiar las trabas y canales que las condiciones coordinativas le imponen y le ofrecen a las invenciones evolutivas no es una tarea simple. La ecuación general de las $C C$ puede ser resuelta, como Whyte (1965) decía, de muchas formas; pero, cada una de ellas impone sus propias restricciones, y abre distintas posibilidades, a los cambios morfológicos y funcionales que la evolución pueda producir. Los constreñimientos y direccionamientos ontogenéticos que rigen la evolución de los vertebrados deben ser muy distintos a los que rigen la evolución de los insectos; $\mathrm{y}$ a ese respecto también deben existir diferencias muy significativas entre mamíferos y batracios. En cada grupo, en cada orden taxonómico, las exigencias de la selección interna son diferentes; pero esto no es muy distinto de lo que ocurre con la selección natural. En rigor, no hay una fuerza única que se llame selección natural: hay un conjunto indefinido y heteróclito de presiones selectivas que actúan de diferente modo sobre diferentes poblaciones, y ellas deben ser individualizadas inductivamente, caso a caso. Cómo también deberán ser individualizadas caso a caso las presiones de la selección interna que actúan en los diferentes taxones.

Claro, insistir en la imagen de un filtro puede reforzar la idea de que la selección interna es sólo un constraint, en el sentido puramente negativo del término, y eso puede desabonar la tesis de que los factores ontogenéticos constituyen comandos o direccionamientos [drives] de la evolución. Pero creo que esta objeción no es aquí pertinente: si la imagen del filtro refuerza la idea de constraint en detrimento de la drive eso también vale para el caso de la selección natural (cf. Dobzhansky, 1983; Martínez, 2007). No hay duda, sin embargo, que este último filtro ha guiado las trayectorias de la evolución; y creo que lo mismo se puede decir de la selección interna: reteniendo sólo lo que es compatible con las condiciones coordinativas está otra fuerza evolutiva no sólo pauta cuáles son las sendas posibles de la evolución; sino que además establece las posibles secuencias de las invenciones evolutivas y hace que algunas de estas sean más probables que otras.

\section{El control de la filogenia por la onto- genia visto desde una perspectiva va- riacional}

Por otro lado, caracterizar a la selección interna como un de filtro, también sirve para dejar más claro un hecho que no me parece de importancia epistemológica menor: el postular un control de la filogenia por parte de la ontogenia no implica el retorno a lo que Elliott Sober (1984) y Richard Lewontin (1985) caracterizarían como una explicación transformacional o desenvolvimiental de la evolución. Al entender el sesgo ontogenético como un resultado de la selección interna estamos subrayando que la Biología Evolucionaria del Desarrollo, conforme lo dije en la presentación, también adopta lo que estos autores definirían como una perspectiva variacional o seleccional que, sin ser asimilable o reducible a la darwiniana, tampoco es incompatible con ella, y, por eso, puede funcionar perfectamente como su complemento.

Recordemos que, conforme Lewontin (1985) y Sober (1984) lo apuntaron, podemos intentar explicar los procesos de cambio que ocurren en cualquier sistema natural o social, apelando a dos tipos de teorías: las desenvolvimientales o transformacionales y las variacionales o seleccionales. Las primeras, nos dicen estos autores, intentan explicar la evolución de un sistema en virtud de cambios simultáneos y conjugados que ocurren en todos y en cada uno de sus componentes. Las segundas, mientras tanto, explican los cambios del sistema en virtud de alteraciones en las proporciones de sus componentes. Estos componentes, se piensa, difieren los unos de los otros en ciertas características, varían; y el conjunto como un todo se modifica a causa de una alteración en la representación proporcional de las diferentes variantes cuyas propiedades específicas permanecen inalteradas (Lewontin, 2000).

Así, como ejemplo paradigmático de explicación transformacional es inevitable citar nuestra concepción de la ontogénesis. Un embrión se desarrolla en virtud de una multitud de cambios paralelos y conjugados que ocurren en las células que lo componen: éstas se multiplican y se diferencian siguiendo determinadas pautas y trayectos; y el resultado total de ese proceso es el desarrollo del organismo. Pueden ser citados, 
con todo, ejemplos de otra naturaleza: si afirmamos que "como grupo, las personas de setenta años son más canosas y más olvidadizas que el conjunto de las que tiene 35 años, porque la mente y el cuerpo de todos los individuos envejecieron", estamos dando una explicación transformacional del estado general de este grupo de personas (Lewontin, 2000). En ambos casos, el sistema total, organismo o grupo humano, se alteró porque así lo hicieron paralelamente todos, o la mayor parte, de sus elementos.

En contrapartida, si decimos que el tamaño medio de una carga de arbejas se incrementó porque fue pasado por un cribo que eliminó las arbejas menores, estamos dando una explicación seleccional de ese cambio: el sistema se modifica porque, por la mediación de un proceso selectivo, se altera la frecuencia y la proporción de sus componentes. Pero estos componentes, se supone, no padecieron individualmente ningún cambio de tamaño. Y el ejemplo de un proceso de cribo es pertinente porque las explicaciones seleccionales suponen siempre alguna suerte de "proceso eliminatorio en el cual algunas variantes persisten en cuanto otras desaparecen, y de ese modo la naturaleza del conjunto cambia sin ningún cambio sucesivo en los elementos individuales" (Lewontin, 1985). Pero, en lo que atañe a nuestro asunto, el mejor modo de entender la distinción entre explicaciones transformacionales y seleccionales es la comparación entre la teoría transformacional de Lamarck y la teoría seleccional de Darwin.

En la teoría de Lamarck, "las especies se alteraban en el tiempo porque cada organismo individual en el interior de la especie sufría los mismos cambios". En esta teoría, como bien lo decía Lewontin (1985), los organismos individuales son "los sujetos de los cambios evolutivos"; es decir: las transformaciones que en ellos ocurren son las que "producen la evolución". Así, aquél que explique esas alteraciones ontogenéticas, podría también explicar el fenómeno evolutivo congregando estas narraciones de procesos individuales de desarrollo en una explicación del fenómeno colectivo. Mientras tanto, "la teoría darwiniana de la evolución orgánica se basa en un modelo variacional" (Lewontin, 2000): en ella el fenómeno evolutivo, ni puede, ni necesita, ser explicado por congregación de narraciones de procesos individuales de transformación. Según esta teoría, una población "se modifica no porque cada individuo pase por desarrollos paralelos durante la vida, y sí porque existe variación entre los individuos y algunas variantes producen más descendientes que otras" (Lewontin, 2000).

Así, y "diferentemente de la historia desenvolvimiental, el enfoque seleccional no explica el hecho de nivel poblacional por agregación de explicaciones individuales" (Sober, 1984). "En lugar de ensamblar narraciones desenvolvimientales individuales en una única explicación de un hecho poblacional", Darwin, nos dice Sober (1984), "planteó la cuestión en un nivel irreductiblemente poblacional". En la teoría lamarckiana, al igual que en cualquiera otra teoría transformacional de la evolución que pueda ser propuesta, lo que en definitiva es explicado, lo que constituye su objetivo explanatorio, es cómo los organismos de un determinado tipo llegaron a tener la forma que de hecho tienen. En la teoría darwiniana, a cambio, lo que se busca explicar es la composición de la población. O dicho de otro modo: mientras la teoría darwiniana explica perfiles poblacionales, las teorías transformacionales explican perfiles orgánicos. En éstas, sería el organismo el que inicial y paulatinamente se modificaría; y la suma y la concatenación de esos fenómenos fisiológicamente verificables explicarían la evolución general de las formas orgánicas. La ontogénesis sería, entonces, la causa de la filogénesis.

Lo interesante, y lo que puede resultar problemático, es que esta última idea parece estar de vuelta. Como vimos más arriba, la Biología Evolucionaria del Desarrollo supone, en efecto, que la ontogenia, de alguna manera, pauta y encausa la filogenia; y esto parece estar implicando el retorno a una explicación desarrollista o transformacional del cambio evolutivo: una explicación contraria al carácter variacional de la teoría darwiniana. La Biología Evolucionaria del Desarrollo, al no olvidar que todo cambio evolutivo debe corporizarse antes en una alteración ontogenética viable, parecería querer explicar los fenómenos evolutivos considerándolos como el agregado o la resultante de cambios ocurridos a nivel del organismo individual; y hasta se podría pensar que es ahí en donde reside la razón más profunda de 
las desavenencias entre los darwinistas ortodoxos y los amigos del desarrollo.

Pero, para que la Biología Evolucionaria del Desarrollo pueda ser considerada como un verdadero retorno al desarrollismo, sería necesario pensar que la sus posiciones presuponen algo semejante a la rehabilitación de una teoría preweismanniana de la herencia; y esto, por supuesto, nadie querría sostenerlo: la transmisión de los caracteres adquiridos, como se quiera que ella sea entendida, no es el mecanismo por el cual la ontogenia incidiría en la filogenia. Nadie está sosteniendo que cambios ocurridos a lo largo de la ontogenia se pueden transmitir a la descendencia, produciendo así un impacto filogenético.

E1 internalismo adoptado por la Biología Evolucionaria del Desarrollo (Azkonobieta, 2005), la idea tan cara a Whyte de que no sólo las exigencias externas o ambientales son las que definen los perfiles de lo viviente, tal vez puede permitirnos hablar de un retorno del organismo (Arthur, 2004a) en la Biología Evolucionaria. Pero lo cierto es que éste no volvió para tener el papel de agente, sujeto, o locus privilegiado de la evolución que tenía en la teoría de Lamarck. Lejos de producir las modificaciones que orientarán la evolución, lejos de instruir a la filogenia, las ontogenias individuales continúan siendo meros ensayos o, si se prefiere, experimentos cruciales, en donde las alternativas de cambio son testadas ya no sólo en virtud de sus ventajas ecológicas, sino también en función de su viabilidad ontogenética. Lo interno, lo organísmico, es ahora una instancia de selección, y no de generación, de las variantes que puedan surgir. La filogenia, tal vez quepa ahora decir, no es más que una serie de experimentos ontogenéticos; y esto queda más claro si ese control de la filogenia por la ontogenia se entiende a la manera de Whyte: se mantiene a la variación hereditaria como la fuente último de innovaciones, pero entre ella y la selección natural se postula un primer filtro: el de la selección interna.

\section{Irreversibilidad y acumulación de los efectos de la selección interna}

Es digno se subrayar, por otra parte, que ese filtro de la selección interna es capaz de producir una secuencia de cambios irreversibles; y esto también puede servirnos para comprender su estatuto de factor director, y no meramente limitante, de la evolución. Cuando la selección interna acepta una reprogramación ontogenética en virtud de su compatibilidad con las condiciones coordinativas; esa circunstancia altera y condiciona sus futuros veredictos sobre otras reprogramaciones; porque, de hecho, el primer cambio ya implica una resolución diferente de dichas condiciones. Así cambios que antes hubiesen sido aceptados ahora no lo serán más; y otros que antes eran imposibles ahora dejarán de serlo. A su vez, claro, la probabilidad o la facilidad de muchas reprogramaciones ontogenéticas que ya antes eran accesibles se verá alterada por la instauración de una nueva resolución de las condiciones coordinativas.

Es decir: si supusiésemos que los dictámenes por sí o por no de la selección interna fuesen siempre recurrentes y constantes, como los de un termostato, se podría decir que ella sólo explica por restricciones, que ella es sólo considerada como un poder limitante o negativo (cf. Bateson, 1973); pero en la medida en que se acepte que cada uno de esos dictámenes sienta una jurisprudencia que alterará y condicionará la naturaleza de las sentencias venideras, la comparación con el termostato ya no cabrá. Los termostatos son sistemas conservadores que se obstinan en preservar un mismo estado privilegiado (Cereijido, 1978); y la selección interna, según la presenta Whyte, puede alterar la definición de esos estados. Y así, bajo este supuesto, las decisiones de esa primera, pero inapelable, corte a la que se someten las alteraciones ontogenéticas pueden ser consideradas como una fuerza capaz de cambiar las vías posibles de la evolución y de empujar a ésta por bretes sin retorno.

Pero creo que hay más: las tesis de Whyte nos permiten pensar que la selección interna, por sí misma, al igual que la selección natural darwiniana (Neander, 1995; Martínez, 2007), pero con independencia de ella, también puede producir efectos acumulativos en los perfiles de los seres vivos. Dichos efectos, claro, no tendrían que ver con las demandas de la lucha por la existencia sino que estarían relacionados con la seguridad y la confiabilidad de los procesos ontogenéticos. El modo más fácil de producir una descendencia capaz de pasar por el filtro de la selección interna es garantizar que la 
información hereditaria esté lo más protegida posible de cualquier perturbación que pueda producir variaciones deletéreas. Dada una población inicial de cualquier tipo de seres vivos, aquellos individuos que se reproduzcan con mayor fidelidad tenderán a tener menos chances de generar criaturas que sean eliminadas por un simple y rotundo no de la selección interna, que aquellos cuya información hereditaria esté más expuesta a ruido capaz de producir mutaciones que generen formas contrarias a las condiciones coordinativas.

Es cierto que, al decir de Whyte, los veredictos de la selección interna no son comparativos: según su perspectiva, los ciclos de desarrollo no son más o menos adecuados a las condiciones coordinativas. Lo son o no lo son. Pero, aun así, se puede pensar que distintos organismos tendrían probabilidades desiguales de producir una descendencia que se adecue a esas condiciones. Puede decirse, por eso, que, aunque sea de un modo indirecto, la selección interna tendría que favorecer cualquier propiedad que torne más estable la transmisión hereditaria; y entre dichas propiedades se podrían encontrar esos mecanismos de reversión mutaciones deletéreas a los que Whyte se refería. El problema, claro, es que esa tendencia conservadora de la selección interna podría también atentar contra la capacidad de evolucionar que caracteriza a los seres vivos. A primera vista, por lo menos, esta la línea de razonamiento nos llevaría a concluir que en términos de selección interna nada sería mejor que una ontogenia blindada contra cualquier perturbación que la pueda alterar: esa sería, claro, la forma más simple de evitar configuraciones contrarias a las condiciones coordinativas. Pero, esa también sería una manera de tornar imposible la evolución.

La selección interna, podríamos pensar, sería una fuerza que, desde el origen de la vida, estaría trabajando en contra de la capacidad de evolucionar. Creo, sin embargo, que es demasiado simplista pensar que el progresivo blindaje de la ontogenia sea la forma por medio de la cual se consiga esa seguridad de los procesos ontogenéticos hacía la que obstinadamente debería tender la selección interna; $y$, para entender por qué, es necesario recordar un aspecto fundamental y desde hace mucho tiempo reconocido de los seres vivos: me refiero a su organización jerárquica. No todas las estructuras y rasgos de los seres vivos tienen la misma importancia organizacional; $\mathrm{y}$ aquellos que son organizacionalmente menos importantes, como Cuvier (1817) ya lo decía cuando enunciaba su Principio de la Subordinación de los Caracteres, pueden variar en mayor grado que aquellos que son organizacionalmente más importantes o dominadores. Así, podemos pensar ahora nosotros, cuando menos importante sea un carácter, más indiferente será la selección interna tanto en lo que toca a sus variaciones como en lo que toca a su variabilidad: ella dejará pasar más variantes de los mismos; y así no tendrá cómo premiar su estabilidad.

Esta última, sin embargo, continuaría siendo premiada en esos caracteres organizacionalmente más importantes que a menudo se configuran en las fases más tempranas de la ontogenia (Arthur, 2004a); y cuánto más importantes sean esos caracteres, mayor sería la propensión a mantener su estabilidad. La constancia evolutiva de las homologías y de los grandes bauplanes podría ser así explicada, por lo menos parcialmente, como un resultado de la selección interna; y al decir esto no estamos más que insistiendo sobre la tesis de que esas estructuras más estables no deben considerarse sólo como el simple remanente de una forma ancestral común que la selección natural habría dejado, por ahora, intocado, sino como el resultado de constreñimientos ontogenéticos que activamente preservan ciertas estructuras en virtud de su importancia organizacional (Hall, 1992; Donoghue, 1992; Raff, 1996; Amundson, 2005; Brigandt, 2007).

Serían los caracteres de importancia secundaría, que resultan más variables, y que en general surgen en etapas tardías de la ontogenia (Arthur, 2004a), los que ofrecerían las alternativas a ser explotadas por la selección natural. Así, la divergencia morfológica seguiría siendo explicada por la intervención de esta última; pero la semejanza ya no sería explicada, por lo menos no exclusivamente, por mera filiación común: ahora también podríamos explicarla como un producto de la selección interna. Pero creo que cabe ir todavía un poco más lejos y afirmarse que la selección interna podría ser pensada como una fuerza capaz de hacer algo más que simplemente dejar un margen de variabilidad a ser explotado por la selección natural. 
Aún como resultado de su inevitable tendencia a incrementar la robustez de los procesos ontogenéticos, ella también podría propiciar, y no sólo tolerar, la evolucionabilidad de las formas orgánicas. Ocurre que el blindaje de una estructura no es el único modo de incrementar su robustez. Sea cual sea el tipo de sistema o de proceso al que nos estemos refiriendo, biológico, social o tecnológico, la rigidez no es el único modo de garantizar su estabilidad; y en general nunca es el más eficiente. Existen otras propiedades organizacionales que la selección interna también podría premiar, y ellas tienen más que ver con la flexibilidad y la complejidad que con la rigidez y la simplicidad. Un ejemplo de ello es la redundancia y otro es la modularidad o compartimentalización organizacional (cfr. Arthur, 2004a; Hammerstein et al., 2005).

Un incremento en la complejidad de un ser vivo producido por la duplicación o, más en general, por la multiplicación, funcional y ontogenéticamente innecesaria, y por eso redundante pero también no deletérea, de una estructura organizacionalmente importante, puede ser también un recurso para incrementar la seguridad de los procesos ontogenéticos (cfr. Arthur, 2004a y 2006). Las ontogenias que incorporasen esas redundancias tolerarían cualquier falencia en la estructura originaria, mucho mejor que aquellas que no las presenten; y, por ello, tendrían más chances de pasar por el filtro de la selección interna.

Pero, además de operar como un reaseguro de la ontogenia, esa redundancia puede también generar un incremento en la capacidad de evolucionar de esos organismos: una vez preservadas por la selección interna, algunas de esas estructuras redundantes pueden ser cooptadas y trabajadas por la selección natural para cumplir con otras funciones (Arthur, 2004a y 2006); y, si esas funciones se transforman, a su vez, en algo organizacionalmente muy importante, se crearán condiciones propicias para que la selección interna también premie su redundancia y para que, a continuación, la selección natural cincele y modifique también algunas de esas nuevas estructuras. La selección interna, podríamos decir, sería capaz de generar un espiral de complejidad y evolucionabilidad creciente.

Claro, para que la selección natural pueda cooptar y trabajar esas estructuras redundantes sería necesario que las modificaciones que ellas vengan a sufrir no afecten el funcionamiento y el desarrollo de sus homólogas. Pero, si es cierto que una correlación demasiado estricta de las partes constituye un obstáculo para la evolución en mosaico producida por selección natural (Gould, 1984; Caponi, 2004); también es cierto que esa correlación demasiado estricta es, ya antes, una propiedad organizacionalmente peligrosa y por eso pasible de ser limitada por la selección interna.

Organismos donde cualquier cambio en una de sus estructuras, o fases de desarrollo, pueda afectar a las demás, son más propensos a sufrir mutaciones deletéreas que aquellos en donde una estructura puede cambiar sin implicar un cambio en el conjunto; y por eso puede decirse que un sistema constituido por módulos o compartimientos funcionales relativamente autónomos será más seguro que uno absolutamente integrado (Moss, 2003; Arthur, 2004a; Azkonobieta, 2005). Así, organismos cuyas estructuras presenten cierto grado de independencia funcional y ontogenética tenderán a pasar más fácilmente el filtro de la selección interna que aquellos que no presenten esa propiedad clave para la evolucionabilidad.

En suma: hay propiedades que la selección interna podría propiciar y acentuar con independencia de la selección natural; y esto justifica a Whyte (1965) cuando decía que la omnipresente vigilancia que las condiciones coordinativas ejercen sobre la ontogenia son capaces, no sólo de controlar, sino también de promover cambios evolutivos aun en ausencia de toda selección natural. En circunstancias muy particulares, en donde por sobreabundancia de recursos disponibles no se da el caso de que nazcan más individuos de los que pueden sobrevivir, la selección natural darwiniana queda en suspenso; y ahí la única fuerza que controlará el cambio evolutivo será la selección interna. Pero, ese control, lejos de limitarse a evitar que surjan variantes contrarias a las condiciones coordinativas, también acabará propiciando esas características que hacen que los procesos ontogenéticos sean más seguros; y esto podrá producir un incremento de la complejidad, y de la variedad, sin que sea preciso atender a las exigencias de la selección darwiniana. Así, cuando la bonanza se acabe, esta última deberá trabajar sobre un conjunto de estructuras y alternativas generadas por la propia selección interna. 


\section{Conclusión}

Lo habitual, lo típico, sin embargo, sería que selección interna y selección natural trabajen simultáneamente: ésta preservando la adecuación del viviente a las exigencias del entorno y aquella preservando su adecuación a condiciones coordinativas. Pero aun así, es posible representar esa división de tareas como una secuencia:

\section{Variación Genética $\rightarrow$ Selección Interna $\Rightarrow$ Variantes fenotípicas $\rightarrow$ Selección Natural}

Se puede conceder, por hipótesis, que la variación genética sea isotrópica como Wallace pretendía, o se puede pensar que ese in-put inicial de cambio está sujeto a ciertas restricciones intrínsecas producidas por la propia arquitectura de los circuitos genéticos (Kauffman, 1993) y por esas capacidades de autoreparación que actuarían en los procesos de replicación. Esas restricciones, sin embargo, no serían suficientes para explicar el sesgo ontogenético que limita la oferta de fenotipos alternativos a ser premiados o castigados por la selección natural: ese sesgo sería producido por la selección interna que, de todas las variaciones efectivamente producidas sólo dejaría pasar aquellas que se ajustan a las condiciones coordinativas.

Pero, independientemente de que pensemos a la variación a la manera de Wallace o la manera de Kauffman, es necesario no olvidar que ella está obviamente restringida por su punto de partida. Una verdad de Perogrullo no deja de ser una verdad: las variaciones a las que está sujeto el genotipo de un burro no son las mismas a las que está sometido el genotipo de un caballo. Por eso, el punto de partida real o efectivo de esa secuencia es siempre muy importante; y esa importancia no sólo afecta la naturaleza de la variación sino que también afecta los dictámenes de la selección interna y de la selección natural. Lo que se aceptará como organizacionalmente $u$ ontogenéticamente viable dependerá de cuál es la configuración que sirve como punto de partida del proceso; y lo que se premie o castigue como ecológicamente ventajoso o desventajoso dependerá también del pico adaptativo ocupado por la población en análisis. La historia de los seres vivos nunca puede ser olvidada y sus marcas también determinan las sendas que la evolución podrá tomar en cualquier coyuntura particular. Esas marcas, sin embargo, también son el decantado de los anteriores dictámenes de la selección interna y la selección natural.

Acada momento de su evolución, las configuraciones de los seres vivos, podemos entonces decir, son la resultante de las exigencias organizacionales o internas, de las exigencias ecológicas o externas y de la propia estructura que esos seres han heredado de sus ancestrales; y esa tríada de codeterminantes de las formas orgánicas podría ser captada en una versión modificada en la letra, pero no en el espíritu, del triangulo exaptativo propuesto por Stephen Jay Gould (2002). Ella sería así:

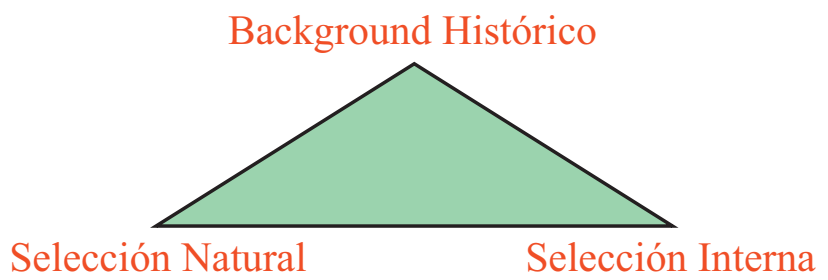

En una teoría completa de la evolución, podríamos decir entonces, los perfiles de los seres vivos no sólo deben ser considerados como respuestas a las exigencias ambientales externas que promueven la selección natural (vértice izquierdo, darwiniano); sino que también deben ser comprendidos en términos de las exigencias de coherencia organizacional que se concretizan en la selección interna (vértice derecho, hoy estudiado por la Biología Evolucionaria del Desarrollo). Pero, es necesario también no olvidar que ambos procesos selectivos tienen siempre como punto de partida el producto acumulado de sus dictámenes anteriores: hay siempre una estructura heredada de un momento anterior que no puede dejar de ser considerada a la hora de entender cualquier configuración efectiva de los seres vivos.

\section{Referencias}

Amundson, R. 2005. The changing role of the embryo in evolutionary thought. Cambridge University Press, Cambridge, UK, 280pp.

Amundson, R. 2001. Adaptation and Development: on the lack of a common ground. In: Orzack, S. \& Sober, E. (eds). Adaptationism and Optimality. Cambridge University Press, Cambridge, UK, p.303-334. 
Arthur, W. 1997. The origin of animal body plans. Cambridge University Press, Cambridge, UK, 339pp.

Arthur, W. 2001. Developmental drive: An important determinant of the direction of phenotypic evolution. Evolution \& Development, 3 (4): 271-278.

Arthur, W. 2004a. Biased Embryos and Evolution. Cambridge University Press, Cambridge, UK, 233pp.

Arthur, W. 2004b. The effect of development on the direction of evolution: toward a twenty-century consensus. Evolution \& Development, 6 (4): 282-288.

Arthur, W. 2006. Creatures of accident. Hill \& Wang, New York, USA, 255pp.

Azkonobieta, T. 2005. Evolución, desarrollo y (auto)organización. Un estudio sobre los principios filosóficos de la EvoDevo. Tesis Doctoral, Universidad del País Vasco San Sebastián, España, 217pp.

Bateson, G. 1973. Steps to an ecology of mind. Paladin, Frogmore, Australia, 510pp.

Bertalanffy, L. 1979 [1953]. Perspectivas en la Teoría General de Sistemas. Alianza, Madrid, España, 166pp.

Bowler, P. 1985. El eclipse del darwinismo. Labor, Barcelona, España, 286pp.

Brigandt, I. 2007. Typology now: homology and developmental constraints explain evolvability. Biology \& Philosophy, 22: 709-725.

Caponi, G. 2004. Los objetivos cognitivos de la paleontología cuvieriana. Principia, 8 (2): 233-258.

Caponi, G. 2005. Funcionalismo cuvieriano vs adaptacionismo darwiniano: consideraciones sobre la noción de condiciones de existencia. Episteme, 22: 79-100.

Cereijido, M. 1978. Orden, equilibrio y desequilibrio. Nueva Imagen, México, México, 224pp.

Cuvier, G. 1817. Le règne animal I. Deterville, Paris, Francia, 539pp.

Cuvier, G. 1992 [1812]. Recherches sur les ossements fossiles de quadrupèdes [Discours préliminaire]. Flammarion, Paris, Francia, 189pp.

Dobzhansky, T. 1983. El azar y la creatividad en la evolución. In: Ayala, F. \& Dobzhansky, T. (eds). Estudios sobre la filosofía de la biología. Ariel, Barcelona, España, p.392-430.

Dobzhansky, T.; Ayala F.; Stebbins, G.; Valentine, J. 1980. Evolución. Omega, Barcelona, España, 558pp.

Donoghue, M. 1992. "Homology". In: Keller, E. \& Lloyd, E. (eds). Keywords in Evolutionary Biology. Harvard University Press, Cambridge, USA, p.170-179.

Goldstein, K. 1951. La structure de l'organisme. Gallimard, Paris, Francia, 446pp.

Gould, S. 1984. Dientes de gallina y dedos de caballo. Blume, Barcelona, España, 415pp.

Gould, S. 2002. The structure of Evolutionary Theory. Harvard University Press, Cambridge, USA, 1433pp.

Grene, M.; Depew, D. 2004. The Philosophy of Biology: An episodic history. Cambridge University Press, Cambridge, UK, 416pp.

Hall, B. 1992. Evolutionary Developmental Biology. Chapman \& Hall, London, UK, 386pp.

Hammerstein, P.; Hagen, E.; Hertz, A.; Herzel, H. 2005. Robustness: a key to evolutionary design. Biological Theory, 1: 90-93.

Huxley, J. 1943. The modern synthesis. Allen \& Unwin, London, UK, 702pp.
Kauffman, S. 1993. The origins of order. Oxford University Press, New York, USA, 709pp.

Keller, E. 2000. The century of the gene. Harvard University Press, Cambridge, USA, 186pp.

Lecointre, G.; Le Guyader, H. 2001. Classification phylogénetique du vivant. Belin, Paris, Francia, 560pp.

Lewontin, R. 1985. The organism as subject and object of evolution. In: Lewontin, R. \& Levins, R. (eds). The dialectical biologist. Harvard University Press, Cambridge, USA, p.84-93.

Lewontin, R. 2000. The triple helix. Harvard University Press, Cambridge, USA, 136pp.

Lewontin, R.; Caspari, E. 1960. Developmental selection of mutations. Science, 132: 1688-1692.

Martínez, M. 2007. La selección natural y su papel causal en la generación de la forma. In: Rosas, A. (ed.). Filosofía, darwinismo y evolución. Universidad Nacional de Colombia, Bogotá, Colombia, p.19-35.

Maynard Smith, J.; Burian, R.; Kauffman, S.; Alberch, P.; Campbell, B.; Goodwin, B.; Lande, R.; Raup, D.; Wolpert, L. 1985. Developmental constraints and Evolution. The Quarterly Review of Biology, 60: 265-287.

Moss, L. 2003. What genes can't do. MIT Press, Cambridge, USA, 228pp.

Neander, K. 1995. Pruning the tree of life. The British Journal for the Philosophy of Science, 46: 59-80.

Raff, R. 1996. The shape of life. The University of Chicago Press, Chicago, USA, 520pp.

Raff, R. 2000. Evo-devo: The evolution of a new discipline. Nature Reviews Genetics, 1: 74-79.

Russell, E. S. 1916. Form and function. Murray, London, UK, 383pp.

Sober, E. 1984. The nature of selection. The Chicago University Press, Chicago, USA, 383pp.

Von Uexkull, J. 1945 [1913]. Ideas para una concepción biológica del mundo. Espasa Calpe, Buenos Aires, Argentina, 276pp.

Wagner, G.; Schwenk, K. 2000. "Evolutionarily stable configurations: Functional integration and the evolution of phenotypic stability". Evolutionary Biology, 31: 155-227.

Wagner, G.; Schwnenk, K. 2003. Constraint. In: Hall, B. \& Olson, W. (eds). Keywords and Concepts in Evolutionary Developmental Biology. Harvard University Press, Cambridge, USA, p.52-60.

Wallace, A. R. 1891. "Creation by law". In: Wallace, A. R. (ed.). Natural Selection and Tropical Nature. Macmillan, London, UK, p.141-166.

Whyte, L. 1960a. Developmental selection of mutations. Science, 132: 954 .

Whyte, L. 1960b. Developmental selection of mutations [réplica a Lewontin, R. \& Caspari, E. 1960]. Science, 132: 1692-1694.

Whyte, L. 1964. Internal factors in evolution. Acta Biotheoretica, 17: 33 .

Whyte, L. 1965. Internal factors in evolution. Braziller, New York, USA, $127 \mathrm{pp}$.

Wilkins, A. 2002. The evolution of developmental pathway. Sinauer, Sunderland, USA, 364pp. 